\title{
702 TJ-CD4B (ABL111), A CLAUDIN18.2-TARGETED 4-1BB TUMOR ENGAGER INDUCES POTENT TUMOR- DEPENDENT IMMUNE RESPONSE WITHOUT DOSE- LIMITING TOXICITY IN PRECLINICAL STUDIES
}

${ }^{1}$ Wenqing Jiang*, 'Zhengyi Wang, 'Zhen Sheng, ${ }^{2}$ Jaeho Jung, ${ }^{1}$ Taylor Guo. ${ }^{1} /-M A B$ Biopharma, Shanghai, China; ${ }^{2}$ ABL Bio, Seongnam, Korea, Republic of

Background 4-1BB (CD137) is a co-stimulatory receptor that stimulates the function of multiple immune cells. Its ability to induce potent anti-tumor activity makes $4-1 \mathrm{BB}$ an attractive target for immuno-oncology. However, clinical development of a monospecific 4-1BB agonistic antibody has been hampered by dose-limiting hepatic toxicities. To minimize systemic toxicities, we have developed a novel Claudin18.2 (CLDN18.2) $\mathrm{x}$ 4-1BB bispecific antibody, TJ-CD4B (ABL111) that stimulates 4-1BB pathway only when it engages with Claudin 18.2, a tumor-associated antigen specifically expressed in gastrointestinal cancers. TJ-CD4B (ABL111) is now being evaluated in patients with advanced solid tumors in a first-in-human trial (NCT04900818).

Methods TJ-CD4B (ABL111) was evaluated in vivo using the human 4-1BB knock-in mice bearing CLDN18.2 expressing MC38 tumor cells. Pharmacodynamic effects upon treatment were characterized in tumor tissue and blood. Immunophenotyping of the tumor microenvironment (TME) and peripheral blood was performed by flow cytometry. Soluble biomarkers were measured using Luminex-based multiplex assay. In-depth gene expression analysis was performed on primary human CD8 + T cells that were co-cultured with CLDN18.2 expressing cells in the presence of anti-CD3 using NanoString nCounter ${ }^{\circledR}$. Pharmacokinetic $(\mathrm{PK})$ and toxicity study were performed in cynomolgus monkeys.

Results TJ-CD4B (ABL111) elicited complete tumor regression in 13 out of 18 MC38 tumor bearing mice given at a dose above $2 \mathrm{mg} / \mathrm{kg}$. Dose-dependent anti-tumor activity was associated with enhanced $\mathrm{T}$ cell activation in TME and expansion of memory $\mathrm{T}$ cells in the peripheral blood. Increased CD8+ $\mathrm{T}$ cells number and proliferation were observed in both tumor nest and surrounding stroma while the level of soluble 4-1BB in the serum was also elevated in response to the treatment. In vitro gene expression analysis by Nanostring revealed TJCD4B(ABL111) effectively activated immune pathways characterized by IFN?-signaling and T cell inflammation. Preclinically, TJ-CD4B was well tolerated at the repeated doses up to 100 $\mathrm{mg} / \mathrm{kg} / \mathrm{wk}$ in cynomolgus monkeys without the adverse influence on the liver function which is generally affected by 4$1 \mathrm{BB}$ activation. Besides, no cytokine release or immune activation was observed in the periphery.

Conclusions TJ-CD4B (ABL111) is a novel CLDN18.2 dependent 4-1BB bispecific agonist antibody that induced $\mathrm{T}$ cell activation and memory response in tumor with CLDN18.2 expression, leading to a strong anti-tumor activity in vivo. TJ-CD4B did not induce systemic immune response nor hepatic toxicity due to the CLDN18.2 dependent 4-1BB stimulation. These data warrant the current clinical development in phase I trial to validate the safety properties and tumor specific responses.

http://dx.doi.org/10.1136/jitc-2021-SITC2021.702 